\title{
Isolation of mutants deficient in acetyl-CoA synthetase and a possible regulator of acetate induction in Aspergillus niger
}

\author{
Heather M. Sealy-Lewis and Valerie Fairhurst \\ Author for correspondence: Heather M. Sealy-Lewis. Tel: +44 1482 465970. Fax: +44 1482465458. \\ e-mail : h.m.sealy-lewis@biosci.hull.ac.uk
}

Department of Biological Sciences, University of Hull, Hull HU6 7RX, UK

\begin{abstract}
Acetate-non-utilizing mutants in Aspergillus niger were selected by resistance to $1.2 \%$ propionate in the presence of $0.1 \%$ glucose. Mutants showing normal morphology fell into two complementation groups. One class of mutant lacked acetyl-COA synthetase but had high levels of isocitrate lyase, while the second class showed reduced levels of both acetyl-CoA synthetase and isocitrate lyase compared to the wild-type strain. By analogy with mutants selected by resistance to $1.2 \%$ propionate in Aspergillus nidulans, the properties of the mutants in A. niger suggest that the mutations are either in the structural gene for acetyl-COA synthetase (acuA) or in a possible regulatory gene of acetate induction (acuB). A third class of mutant in a different complementation group was obtained which had abnormal morphology (yellow mycelium and few conidia); the specific lesion in these mutants has not been determined.
\end{abstract}

Keywords: acetyl-CoA synthetase, propionate resistance, Aspergillus niger

\section{INTRODUCTION}

It has previously been reported in Aspergillus nidulans that spontaneous resistant sectors arose on $1.2 \%$ propionate medium, some of which were acetate-nonutilizing mutants, and it was suggested that propionate could be used as a general selection technique for isolating acetate-non-utilizing mutants in filamentous fungi (Sealy-Lewis, 1994). Three classes of acetate-nonutilizing mutant were obtained, in (i) $f a c A(a c u A)$, which encodes the structural gene for acetyl-CoA synthetase (ACS) (Apirion, 1965; Armitt et al., 1976; Sandeman \& Hynes, 1989), (ii) facB (acuB), a regulatory gene involved in the induction of ACS, enzymes of the glyoxylate bypass, acetamidase and NADP-isocitrate dehydrogenase (Apirion, 1965; Armitt et al., 1976; Kelly \& Hynes, 1977, 1982; Katz \& Hynes, 1989; Todd et al., 1997) and (iii) acuO, a gene which had not previously been described and whose function is unknown. At least two of the classes of mutant selected by resistance to propionate were also selected as being resistant to fluoroacetate ( $f a c A$ and $f a c B$ mutants; Apirion, 1965). acu $\mathrm{O}$ has not been tested on fluoroacetate as a substrate. Although no mutants in $\mathrm{facC}$ were isolated as being

Abbreviations: ACS, acetyl-CoA synthetase; ICL, isocitrate lyase. resistant to propionate, a previously isolated facC102 mutant is also resistant to propionate (unpublished). In addition to the mutants described above, a mutant was also isolated that was resistant to propionate but was not defective in acetate metabolism; this was found to be either allelic with, or closely linked to $f a c A$. A general screen for propionate-resistant mutants in $A$. nidulans resulted in $\mathrm{Acu}^{-}$and $\mathrm{Acu}^{+}$isolates with approximately equal frequency (unpublished); the latter class of mutant could be analogous to the fluoroacetate-resistant $\mathrm{Acu}^{+}$ mutants (fan mutants) isolated by Armitt et al. (1976).

The basis of the selection on propionate of the acetatenon-utilizing mutants was thought to be that propionate itself is not toxic, but is converted to a toxic product. Propionate is metabolized by the 2-methylcitrate pathway in Saccharomyces cerevisiae (Pronk et al., 1994); this pathway was also found in 21 yeast species and six species of filamentous fungi when surveyed by Miyakoshi et al. (1987). The initial step of this pathway is the formation of 2-methylcitrate from propionyl-CoA and oxaloacetate. In a series of reactions similar to the normal tricarboxylic acid cycle reactions, 2-methylcitrate is converted to 2-methylisocitrate, which is then cleaved into pyruvate and succinate. Much less commonly, propionate was found to be metabolized through a modified $\beta$-oxidation pathway. Plants, humans and 
insects utilize the methylmalonyl-CoA pathway (Wegener et al., 1968; Rosenberg \& Fenton, 1989; Halarknar et al., 1985). The enzymes of the 2-methylcitrate pathway have been found in Aspergillus niger, but have not been confirmed in A. nidulans (Miyakoshi et al., 1987). Whichever pathway is utilized, the initial step involves the conversion of propionate to propionylCoA. Thus, $f a c A$ and $f a c B$ mutants are resistant to propionate because they lack ACS. facC has recently been cloned and sequenced (Stemple, 1997) and encodes a cytoplasmic acetylcarnitine transferase. The role of acu $\mathrm{O}$ is still unknown.

A. nidulans is generally regarded as a model organism for studies on the molecular genetics and biochemistry of filamentous fungi. We thought that it would be of interest to use the propionate selection technique to identify mutations in comparable steps in acetate utilization in the commercially important $A$. niger and establish whether the regulation of the early steps of acetate activation is similar in the two organisms.

Selection for resistance to sodium propionate as the sole carbon source in $A$. niger did not yield any resistant mutants. However, in contrast to A. nidulans, $A$. niger was unable to grow on $1.2 \%$ propionate in the presence of $0.1 \%$ glucose, and acetate-non-utilizing mutants were obtained on this medium. We present evidence for the isolation of mutants in three complementation groups, including probable mutations in the structural gene for $\mathrm{ACS}$ and a possible regulatory gene.

\section{METHODS}

Strains and genetic analysis. The wild-type strain was $\operatorname{csp} A 1$; nicA1 having short conidiospores and a requirement for nicotinic acid. The strain used for haploidization analysis was N827: fwA1; bisD4; bioA1 lysA7; leuA1; pabA1; cnxC5; $\operatorname{trpB2}$ (kindly provided by Dr Klaas Swart, Department of Genetics, Wageningen Agricultural University, NL). The markers are described by Bos et al. $(1988,1993)$. The medium on which $A$. niger was grown was the same as that described for A. nidulans by Pontecorvo et al. (1953) and McCully \& Forbes (1965), and modified by Cove (1966). The method used for assigning markers to linkage groups has been described by Bos et al. (1988, 1993).

Selection of mutants resistant to propionate. After UV mutagenesis, conidia were plated onto medium containing $1.2 \%(\mathrm{w} / \mathrm{v})$ sodium propionate with or without $0.1 \%(\mathrm{w} / \mathrm{v})$ glucose.

Growth of mycelia for enzyme assays. Mycelia were grown in shaking cultures for $18 \mathrm{~h}$ at $37^{\circ} \mathrm{C}$ on $20 \mathrm{mM}$ sucrose, $5 \mathrm{mM}$ urea medium with appropriate supplementation. After filtration, mycelia were transferred for a further $6 \mathrm{~h}$ to medium containing $100 \mathrm{mM}$ sodium acetate (induced) or $50 \mathrm{mM}$ glucose (glucose repressed) as described by Armitt et al. (1976). Extracts were made by grinding the mycelium with sand before the addition of extraction buffer (Armitt et al., 1976) and the extracts were centrifuged at an average of $20000 \mathrm{~g}$ for $30 \mathrm{~min}$.

Enzyme assays. The acetyl-CoA synthetase assay was modified after the method of Berg (1956). Crude extracts were prepared in $40 \mathrm{mM}$ potassium phosphate buffer, $\mathrm{pH} 7 \cdot 4$. Each $600 \mu \mathrm{l}$ reaction contained : $10 \mathrm{mM}$ ATP, $0 \cdot 1 \mathrm{mM}$ coenzyme A, $200 \mathrm{mM}$ hydroxylamine hydrochloride, $10 \mathrm{mM}$ magnesium chloride and $10 \mathrm{mM}$ potassium acetate in $40 \mathrm{mM}$ potassium phosphate buffer, $\mathrm{pH} 7 \cdot 4$. The reaction was started by the addition of 15-120 $\mu$ l crude extract and allowed to proceed for 20-40 min before the reaction was stopped by the addition of $900 \mu \mathrm{l}$ acidified ferric chloride solution. Precipitated protein was removed by centrifugation and the absorbance was measured at $540 \mathrm{~nm}$.

Isocitrate lyase was assayed according to the method of Armitt et al. (1976). The protein concentration was estimated by the microbiuret method (Goa, 1953).

\section{RESULTS}

\section{Isolation of mutants resistant to propionate and glucose}

UV irradiated conidia ( $70 \%$ kill) from the $\operatorname{csp} A 1$; nicA1 strain were plated onto minimal medium with $1.2 \%$ sodium propionate as the sole carbon source as well as onto medium containing $0.1 \%$ glucose $+1.2 \%$ propionate. No resistant colonies were obtained on the propionate medium (total $1.9 \times 10^{8}$ viable conidia screened), but on the propionate +glucose medium, resistant colonies arose with a frequency of 1 in $4.7 \times 10^{5}$ viable conidia. One hundred and forty mutants were originally isolated; these were classified into three groups on the basis of their morphology on complete medium. Class I mutants had a morphology that was indistinguishable from the wild-type strain; the majority of resistant mutants were of this type. The remaining two classes (comprising a total of eight mutants) had abnormal morphology compared to the wild-type; these abnormalities resulted in sparse or no conidiation and a strong yellow colouration of the mycelium. Forty-eight mutants (42 of normal morphology and six of abnormal morphology) were purified so that isolates arose from a single conidium on fresh propionate + glucose medium; these purified isolates were further tested at $37^{\circ} \mathrm{C}$ on medium containing $1.2 \%$ sodium acetate and $5 \mathrm{mM}$ urea. All the mutants grew less well than the wild-type on acetate medium, although in the case of the mutants with morphological abnormalities and where conidiation was poor, it was difficult to assess the degree of impairment. A. niger, in common with A. nidulans, shows very weak growth on agar plates in the absence of any added carbon source. Armitt et al. (1976) concluded that for $A$. nidulans, the carbon source in the agar was lipid or compounds metabolized via $\mathrm{C}_{2}$ units, since this growth was abolished in most of their $\mathrm{Acu}^{-}$strains. On agar medium lacking a carbon source, the diameter of the colony increases at about the same rate as when grown on glucose medium, but the density of the colony is drastically reduced and there is no conidiation. The growth of the class I propionate-resistant mutants was no better on acetate medium than on medium lacking a carbon source.

Twenty-five of the morphologically normal class of mutant and six morphologically abnormal mutants were 
Table 1. Classification of propionate-resistant mutants

\begin{tabular}{|c|c|c|c|}
\hline Class/morphology & Mutant* & Gene designation & $\begin{array}{l}\text { Linkage } \\
\text { group }\end{array}$ \\
\hline $\begin{array}{l}\text { Class I; wild-type } \\
\text { morphology }\end{array}$ & $\begin{array}{l}1.1,7.1,42.1,43.1,45.1, \\
47.1,49.1,50.1,98.1, \\
105.1,127.1,131.1,206.1, \\
213.1,214.1,220.1,223.1, \\
235.1,241.1,251.1,256.1, \\
258.1\end{array}$ & $\begin{array}{l}\text { acuB; fails to complement } \\
98.1\end{array}$ & VIII \\
\hline $\begin{array}{l}\text { Class I; wild-type } \\
\text { morphology }\end{array}$ & $117.1,264.1,255.1$ & acuA; complements 98.1 & Unknown \\
\hline $\begin{array}{l}\text { Class II†; pale yellow } \\
\text { mycelium, very few } \\
\text { conidia }\end{array}$ & $90.1,(129.1)$ & $\begin{array}{l}a c u C ; 90.1 \text { complements } \\
98.1 \text { and } 117.1\end{array}$ & $\mathrm{~V}$ \\
\hline $\begin{array}{l}\text { Class III†; bright } \\
\text { yellow mycelium, no } \\
\text { conidia }\end{array}$ & (133.1), (134.1), 62.1, 91.1 & $\begin{array}{l}a c u D ? 62.1 \text { and } 91.1 \\
\text { complement } 98.1 \text { and } \\
117.1 ; \text { may be the same } \\
\text { gene as } 90.1\end{array}$ & Unknown \\
\hline
\end{tabular}

* The mutants in parentheses are assigned to a group on the basis of their morphology, but complementation tests have not been performed.

† Class II mutants show pale yellow mycelia after $2 \mathrm{~d}$ incubation at $37^{\circ} \mathrm{C}$. Conidiation occurs slowly if left on the bench. Class III mutants show bright yellow mycelia and no conidia at $37^{\circ} \mathrm{C}$ after $2 \mathrm{~d}$ incubation; a few scattered conidia are formed after prolonged incubation on the bench.

\section{Table 2. Growth properties of the wild-type and propionate-resistant mutants}

Growth is represented on a scale of $1-5$. Growth scores are not necessarily comparable between plates. The growth media were: CM (complete medium); SC ( $1 \%$ glucose); $-\mathrm{C}$ (no carbon source); Ac (1.2\% acetate); Prop (1.2\% propionate); Prop + G (1.2\% propionate $+0.1 \%$ glucose); Eth $(1 \%$ ethanol); But $(1.2 \%$ butyrate $)$ and Tween $(0.5 \%)$. In all cases the nitrogen source was $5 \mathrm{mM}$ urea.

\begin{tabular}{|lccccccccc|}
\hline Strain & \multicolumn{8}{c|}{ Growth on: } \\
\cline { 2 - 9 } & CM & SC & - C & Ac & Prop & Prop + G & Eth & But & Tween \\
\hline acuB98.1 & 5 & 5 & $1-$ & $1-$ & 0 & $1^{*}$ & 1 & 1 & 4 \\
acuA117.1 & 5 & 5 & $1-$ & $1-$ & 0 & $1^{*}$ & 1 & 1 & 4 \\
acuC90.1 & 5 & 5 & 1 & 2 & 0 & $1^{*}$ & $1+$ & 0 & 3 \\
acuD91.1 & 3 & 3 & 1 & 2 & 0 & $1^{*}$ & 2 & 0 & 2 \\
Wild-type & 5 & 5 & 1 & 3 & 0 & 0 & $2+$ & 1 & 4 \\
\hline
\end{tabular}

*Growth on Prop $+\mathrm{G}$ was inhibited (colony diameters were reduced) but is indicated as 1 .

selected for detailed analysis by complementation tests (see below) and further growth tests (Tables 1 and 2). The selected mutants were tested on the following carbon sources: $1.2 \%$ propionate, $1.2 \%$ propionate + $0 \cdot 1 \%$ glucose, $1.2 \%$ sodium acetate, $1 \%$ ethanol, $0.5 \%$ Tween 80 (polyoxyethylenesorbitan monooleate), $0 \cdot 1 \mathrm{M}$ butyrate, and on medium lacking a carbon source (Table 2). In each case the nitrogen source was $5 \mathrm{mM}$ urea. None of the mutants showed any growth on propionate medium in the absence of glucose (not even the weak growth seen on medium lacking a carbon source). Growth of the propionate-resistant mutants selected on propionate + glucose was poor, but since there was no growth at all of the wild-type, the colonies were easy to identify in the original screen. This growth can be best interpreted as relief of the toxic effects of propionate in the medium, allowing utilization of the glucose. All the mutants grew less well than the wild-type on ethanol medium, although the mutants in class III $(91.1,62.1$, 133.1 and 134.1) showed the strongest growth (Table 2). The class I mutants (which comprised two genes, see below) grew as well as the wild-type on Tween 80 , but the class II and III mutants showed some growth reduction. Butyrate was a very poor carbon source for 
the wild-type and growth was only observed after a week of incubation; the class II and III mutants did not grow on butyrate, while class I mutants grew as well as the wild-type. In general, the morphological mutants were hard to work with because it proved difficult to construct diploids for haploidization/complementation analysis.

\section{Characterization of mutants}

All the mutants described below were recessive to the wild-type $\mathrm{Acu}^{+}$strain in a diploid with the multimarked strain, N827. A diploid between one of the class I mutants, 98.1, and N827 was haploidized and acu98.1 was found to map to linkage group VIII (data not shown). A recombinant isolate from the haploidization 98.1: N827 (fwA1; bioA1 lys A7; leuA1; cnxC5; acu98.1) was used for complementation tests with the other $\mathrm{Acu}^{-}$ mutants isolated. Complementation tests were performed on acetate medium and propionate + glucose medium. All the putative diploids isolated for these tests were checked to make sure that the volume of the conidia was characteristic of the diploid (see Bos et al., 1988, 1993).

The majority of the mutants failed to complement mutant 98.1 (i.e. they failed to grow on acetate medium and were resistant on propionate + glucose medium) (Table 1). Another mutation of this complementation group, acu105.1, was assigned to linkage group VIII on the basis of haploidization analysis with N827. The mutants tested that complemented 98.1 were 117.1, 264.1 and 255.1, which were class I mutants with no morphological abnormalities. Mutants 90.1, 62.1 and 91.1 also complemented the 98.1 tester strain. It was not possible to make diploids with 129.1, 133.1 and 134.1 and therefore it has not been possible to characterize these mutants. Diploids were made between 117.1 and N827, 90.1 and N827, and 91.1 and N827. Subsequent haploidization analysis assigned acu90.1 to linkage group V, although, presumably because of the extreme morphology associated with acu90.1, no $\mathrm{Acu}^{-}$ segregants were isolated after haploidization. Repeated analyses of segregants from the 117.1:N827 and 91.1: N827 diploids failed to assign these $\mathrm{Acu}^{-}$mutants to a linkage group. However, for both 91.1 (bioA1 lysA7; pabA1 cnxC5 acu91.1) and 117.1 (bioA1 lysA7; $\operatorname{trpB2}$; acu117.1), recombinant genotypes were isolated from the haploidizations that were utilized in further complementation analyses. Mutant 117.1 failed to complement 255.1 and 264.1, making it likely that these contain mutations in the same gene. We were aware that the complementation between acu98.1 and acu117.1 could have been a result of inter-allelic complementation and we therefore looked at complementation of acu117.1 with other alleles in the class that failed to complement acu98.1, namely acu50.1 and acu105.1; again complementation was seen, so we believe that the observed effect is unlikely to be due to inter-allelic complementation. Because A. niger lacks a sexual cycle, it is not possible to confirm by a cross to the wild-type that the $\mathrm{Acu}^{-}$mutant phenotype is the result of a single
Table 3. Relative specific activities of ACS and ICL in wild-type and $\mathrm{ACu}^{-}$mutant strains

All activities are expressed relative to the wild-type strain transferred from $0.02 \mathrm{M}$ sucrose (Suc) to $100 \mathrm{mM}$ acetate medium (Ac) or to $50 \mathrm{mM}$ glucose (Glc). All determinations were performed at least twice, and standard error values are shown. NT; not tested. The wild-type activity induced on acetate (six determinations) was equivalent to a specific activity of $192.4 \mathrm{nmol}$ product $\mathrm{min}^{-1}$ (mg protein) ${ }^{-1}( \pm 9.9)$ for ACS and $260 \cdot 3 \pm 8 \cdot 5 \mathrm{nmol}$ product $\mathrm{min}^{-1} \mathrm{mg}^{-1}$ for ICL.

\begin{tabular}{|c|c|c|c|}
\hline \multirow[t]{2}{*}{ Strain } & \multirow[t]{2}{*}{ Transfer } & \multicolumn{2}{|c|}{$\begin{array}{c}\text { Relative specific activity } \\
(\%)\end{array}$} \\
\hline & & ACS & ICL \\
\hline Wild-type & $\mathrm{Suc} \rightarrow \mathrm{Ac}$ & 100 & 100 \\
\hline Wild-type & $\mathrm{Suc} \rightarrow \mathrm{Glc}$ & $11 \cdot 1 \pm 0 \cdot 26$ & $2.7 \pm 0.7$ \\
\hline aсuB98.1 & Suc $\rightarrow$ Ac & $45 \cdot 3 \pm 2 \cdot 9$ & $22 \cdot 5 \pm 1 \cdot 0$ \\
\hline acuB50.1 & Suc $\rightarrow$ Ac & $50.7 \pm 10 \cdot 6$ & $27 \cdot 0 \pm 3 \cdot 4$ \\
\hline acuA117.1 & $\mathrm{Suc} \rightarrow \mathrm{Ac}$ & $4.7 \pm 1.97$ & $89.7 \pm 6 \cdot 2$ \\
\hline acuA255.1 & Suc $\rightarrow$ Ac & $1.7 \pm 0.58$ & $70 \cdot 5 \pm 4 \cdot 5$ \\
\hline acuA264.1 & $\mathrm{Suc} \rightarrow \mathrm{Ac}$ & $2 \cdot 2 \pm 0 \cdot 5$ & $\mathrm{NT}$ \\
\hline асиС90.1 & Suc $\rightarrow$ Ac & $85 \cdot 8 \pm 3 \cdot 75$ & $59 \cdot 0 \pm 4.5$ \\
\hline acuD91.1 & $\mathrm{Suc} \rightarrow \mathrm{Ac}$ & $28 \cdot 2 \pm 3 \cdot 7$ & $37 \cdot 3 \pm 5 \cdot 0$ \\
\hline
\end{tabular}

gene mutation. However, in the haploidization analyses, the propionate-resistant phenotype always segregated with the $\mathrm{Acu}^{-}$phenotype.

It is consistent with the biochemical data (see below) that the complementing groups exemplified by acu 98.1 and $a c u 117.1$ are mutations in different genes. acu117.1 complemented acu90.1, acu91.1 and acu62.1. It has not been possible to construct the appropriate diploids in pairwise combinations between mutants 90.1,91.1 and 62.1 to look at the complementation between these $\mathrm{Acu}^{-}$ mutants. All the mutants involved have morphological abnormalities which fall into two classes, although we cannot say whether they are all mutations in the same gene. They are, however, distinct from the complementing groups represented by acu98.1 and acu117.1. Our data suggest that the $\mathrm{Acu}^{-}$mutants isolated represent mutations in at least three genes.

\section{Assay of enzyme activities in Acu- mutants}

Mycelia grown from mutants in the representative classes together with the wild-type control were grown on sucrose medium and transferred to $100 \mathrm{mM}$ sodium acetate medium or $50 \mathrm{mM}$ glucose medium. Extracts made from the mycelium were assayed for ACS and ICL (one of the enzymes of the glyoxylate bypass) activity (Table 3).

\section{DISCUSSION}

We have previously isolated mutants of $A$. nidulans in $f a c A$ (defective in ACS) and $f a c B$ (regulatory gene involved in acetate induction) by selection for resistance 
to $1.2 \%$ propionate. In $A$. niger, analogous mutants were not selected by this technique, but were obtained after selection on $1.2 \%$ propionate $+0.1 \%$ glucose medium. In A. nidulans, the wild-type strain was able to grow on propionate +glucose medium, presumably because ACS is completely repressed in the presence of glucose (Kelly \& Hynes, 1977; Sandeman \& Hynes, 1989 ) and the wild-type is therefore insensitive to the toxic effects of propionate. In contrast, A. niger shows quite high levels of ACS in the presence of $0.1 \%$ glucose (Table 3 ) and therefore propionate would be converted to its toxic product, and the wild-type would be sensitive on propionate +glucose medium. The reason for the inability to select $A$. niger mutants resistant to $1 \cdot 2 \%$ propionate is not clear.

The $A$. niger mutants that were selected on propionate + glucose medium were divided into four classes on the basis of their morphology and complementation tests; these represent mutations in at least three genes (Table 1). The mutants of the class which includes acuA117.1 lack ACS activity, but have high levels of ICL (Table 3). These mutants are likely to be affected in the structural gene for ACS; we have called this class of mutant $a c u A$. The fact that the $A$. niger $\mathrm{ACS}^{-}$ mutants have high levels of ICL suggests that acetate is the natural inducer, a situation that has been previously reported for A. nidulans (Hynes, 1977) and Aspergillus terreus (Das \& Sen, 1983). The largest class of mutant, of which acuB98.1 is a member, has reduced levels of ACS and ICL and is more similar to the regulatory mutant, $f a c B$, described in $A$. nidulans. We have called this class of mutant $a c u B$. However, the $a c u B$ mutants in A. niger show quite high levels of ACS and ICL and we can only conclude that other enzymes in the glyoxylate pathway are more severely affected or that the levels of enzymes involved in growth on acetate have to be high to sustain growth on this poor carbon source. It has been shown that $A$. niger contains a regulatory gene of similar function to the $A$. nidulans $f a c B$ gene, since the cloned $f a c B$ gene has been isolated from $A$. niger by nucleic acid hybridization, using the $A$. nidulans $f a c B$ gene as a probe. The $A$. niger $f a c B$ homologue can substitute for the $A$. nidulans $f a c B$ function in a null $f a c B$ mutant in $A$. nidulans (Todd et al., 1997). It had previously been reported that although $A$. niger does not contain a gene for acetamidase (amdS), the amdS gene from $A$. nidulans can function in $A$. niger and is inducible by acetate in a $f a c B$-dependent manner, suggesting that the $f a c B$ homologue is required for acetate induction (Davis et al., 1988).

The other mutants isolated, acuC90.1 and acuD91.1, which may be mutations in the same gene, produce gross morphological alterations; because of this it is difficult to say whether they are really unable to grow on acetate. acu91.1 shows impaired levels of ACS and ICL compared with the wild-type strain, while acu 90.1 shows quite high levels of ACS but lower levels of ICL (Table 3). Both mutants fail to grow on medium containing butyrate but they show some growth on medium containing Tween 80 (Table 2). The behaviour of these strains could simply be a result of their aberrant morphology. Both acuC90.1 and acuD91.1 show yellow pigmentation of the mycelium and sparse conidiation, a property that has been described for mutations in $c r e A$ from $A$. niger that map on linkage group IV (Ruijter $e t$ al., 1997) and was thought to be due to derepression of the biosynthesis of a secondary metabolite. The $a c u A$ and $a c u B$ mutants, at least, grow on fatty acids and so their lesions are unlikely to be in the glyoxylate cycle, which would be required for fatty acid breakdown (Kawasaki et al., 1995).

No propionate-resistant mutants that were able to grow on acetate but which had normal morphology were isolated in A. niger (analogous to the fan mutants in $A$. nidulans). In a general screen for mutants resistant to propionate in A. nidulans after UV mutagenesis, of 19 resistant mutants selected at random, 12 were able to grow on acetate medium and seven were $\mathrm{acu}^{-}$mutants (unpublished). If the class of mutant that is propionate resistant but able to grow on acetate occurred with the same frequency in $A$. niger, we should have isolated such mutants. The inability to isolate $\mathrm{Acu}^{-}$mutants on propionate alone and the failure to isolate propionateresistant $\mathrm{Acu}^{+}$mutants seems to be a fundamental difference between $A$. nidulans and $A$. niger. Propionate is a well-known antimicrobial compound with a long history of use as a preservative in foods and pharmaceuticals, although its exact mode of action is not fully understood (Russell \& Chopra, 1990). The toxic intermediate that is responsible for inhibition in $A$. niger and $A$. nidulans cannot be determined from this study, but the concentration of propionate used to isolate mutants is high $(125 \mathrm{mM})$ and as propionate is a substrate for ACS, high levels of propionyl-CoA are likely to accumulate. In Neurospora crassa, acetyl-CoA is known to be toxic during growth on high concentrations of acetate, since strains that lack acetyl-CoA hydrolase fail to grow, although growth is normal on ethanol or on low concentrations of acetate (Connerton et al., 1992). If propionyl-CoA builds up in the mycelium, this might easily be toxic to the cell, either because it cannot be converted to propionate and CoA by the hydrolase or because the propionyl-CoA might inhibit any acetyl-CoA-dependent enzymes essential for growth.

Chattopadhyay et al. (1980) isolated acetate-nonutilizing mutants in $A$. niger after mutagenesis and replica plating onto acetate medium, and obtained two classes of mutant, neither of which corresponds to the mutants selected in this study. It seems likely that the selection method using propionate is widely applicable to filamentous fungi, although it may be necessary to include glucose in the selection medium for some fungal species. A. niger does not have a sexual cycle, but using the parasexual cycle it has been possible to obtain recombinant $a c u$ mutant genotypes and assign mutants to complementation groups. The isolation of acetaterequiring mutants in $A$. niger will aid comparative studies on the regulation of acetate utilization in $A$. nidulans and $A$. niger. 


\section{ACKNOWLEDGEMENTS}

We thank Klaas Swart for providing the A. niger strain N827. We would also like to thank Rory Weber, Samantha Brookes and Helena Smith, who contributed to this work as part of third-year projects.

\section{REFERENCES}

Apirion, D. (1965). The two way selection of mutants and revertants in respect of acetate utilisation and resistance to fluoroacetate in Aspergillus nidulans. Genet Res 6, 317-329.

Armitt, S., McCullough, W. \& Roberts, C. F. (1976). Analysis of acetate non-utilising (acu) mutants in Aspergillus nidulans. J Gen Microbiol 92, 263-282.

Berg, P. (1956). Acyl adenylates: an enzymatic mechanism of acetate activation. J Biol Chem 222, 991-1013.

Bos, C. J., Debets, A. J. M., Swart, K., Huybers, A., Kobus, G. \& Slakhorst, S. M. (1988). Genetic analysis and construction of master strains for assignment of genes to six linkage groups in Aspergillus niger. Curr Genet 14, 437-443.

Bos, C. J., Slakhorst, S. M., Debets, A. J. M. \& Swart, K. (1993). Linkage group analysis in Aspergillus niger. Appl Microbiol Biotechnol 38, 742-745.

Chattopadhyay, P., Banerjee, S. K., Chakrabarti, P. \& Sen, K. (1980). Biochemical studies on acetate non-utilizing $(a c u)$ mutants of Aspergillus niger. Indian J Exp Biol 18, 764-766.

Connerton, I. F., McCullough, W. \& Fincham, J. R. S. (1992). An acetate-sensitive mutant of Neurospora crassa deficient in acetylCoA hydrolase. J Gen Microbiol 138, 1797-1800.

Cove, D. J. (1966). The induction and repression of nitrate reductase in the fungus Aspergillus nidulans. Biochim Biophys Acta 113, 51-56.

Das, T. K. \& Sen, K. (1983). Studies on the control of enzymes for the glyoxylate cycle in Aspergillus terreus. Curr Microbiol 9, 55-58.

Davis, M. A., Cobbett, C. S. \& Hynes, M. J. (1988). An amdS-lacZ fusion for studying gene regulation in Aspergillus nidulans. Gene 63, 199-212.

Goa, J. (1953). A microbiuret method for determination of total protein in cerebrospinal fuid. Scand J Clin Lab Invest 5, 218-222.

Halarnkar, P. P., Nelson, J. H., Heisler, C. R. \& Blomquist, G. J. (1985). Metabolism of propionate to acetate in the cockroach Periplaneta americana. Arch Biochem Biophys 236, 526-534.

Hynes, M. J. (1977). Induction of acetamidase of Aspergillus nidulans by acetate metabolism. J Bacteriol 131, 770-775.

Katz, M. E. \& Hynes, M. J. (1989). Isolation and analysis of the acetate regulatory gene, $f a c B$, from Aspergillus nidulans. Mol Cell Biol 9, 5696-5701.

Kawasaki, L, Farres, A. \& Aguirre, J. (1995). Aspergillus nidulans mutants affected in acetate metabolism isolated as lipid nonutilizers. Exp Mycol 19, 81-85.

Kelly, J. M. \& Hynes, M. J. (1977). Increased and decreased sensitivity to carbon catabolite repression of enzymes of acetate metabolism in mutants of Aspergillus nidulans. Mol Gen Genet 156, 87-92.

Kelly, J. M. \& Hynes, M. J. (1982). The regulation of NADP-linked isocitrate dehydrogenase in Aspergillus nidulans. J Gen Microbiol 128, 23-28.

McCully, K. S. \& Forbes, E. (1965). The use of $p$-fluorophenylalanine with master strains of Aspergillus nidulans for assigning genes to linkage groups. Genet Res 6, 352-359.

Miyakoshi, S., Uchiyama, T., Someya, T., Satoh, T. \& Tabuchi, T. (1987). Distribution of the methylcitric acid and- $\beta$-oxidation pathway for propionate catabolism in fungi. Agric Biol Chem 51, 2381-2387.

Pontecorvo, G., Roper, J. A., Hemmons, L. M., MacDonald, K. D. \& Bufton, A. W. J. (1953). The genetics of Aspergillus nidulans. Adv Genet 5, 141-238.

Pronk, J. T., van der Linden-Beuman, A., Verduyn, C., Scheffers, W. A. \& van Dijken, J. P. (1994). Propionate metabolism in Saccharomyces cerevisiae: implications for the metabolon hypothesis. Microbiology 140, 717-722.

Rosenberg, L. E. \& Fenton, W. A. (1989). Disorders of propionate and methylmalonate metabolism. In The Metabolic Basis of Inherited Disease, 6th edn, pp. 822-844. Edited by C. R. Scriver, A. L. G. Beaudet, W. S. Sly \& D. Valle. New York: McGrawHill.

Ruijter, G. J. G., Vanhanen, S. A., Gielkens, M. M. C., van de Vondervoort, P. J. I. \& Visser, J. (1997). Isolation of Aspergillus niger creA mutants and effects of the mutations on expression of arabinases and L-arabinose catabolic enzymes. Microbiology 143, 2991-2998.

Russell, A. D. \& Chopra, I. (1990). Antiseptics, disinfectants and preservatives: their properties, mechanisms of action and uptake into bacteria. In Understanding Antibacterial Action and Resistance, pp. 95-131. Edited by A. D. Russell \& I. Chopra. Chichester: Ellis Horwood.

Sandeman, R. A. \& Hynes, M. J. (1989). Isolation of the facA (acetyl-Coenzyme A synthetase) and $a c u E$ (malate synthase) genes of Aspergillus nidulans. Mol Gen Genet 218, 87-92.

Sealy-Lewis, H. M. (1994). A new selection method for isolating mutants defective in acetate utilisation in Aspergillus nidulans. Curr Genet 25, 47-48.

Stemple, C. (1997). Analysis of the facC gene of Aspergillus nidulans. MSc thesis, University of Melbourne.

Todd, R. B., Murphy, R. L., Martin, H. M., Sharp, J. A. , Davis, M., Katz, M. E. \& Hynes, M. J. (1997). The acetate regulatory gene $f a c B$ of Aspergillus nidulans encodes a $\mathrm{Zn}$ (II)2Cys6 transcriptional activator. Mol Gen Genet 254, 495-504.

Wegener, W. S., Reeves, H. C., Rabin, R. \& Ajl, S. J. (1968). Alternative pathways of metabolism of short-chain fatty acids. Bacteriol Rev 32, 1-26.

Received 12 December 1997; revised 9 February 1998; accepted 19 March 1998. 